\title{
Ultrabroadband terahertz conductivity of Si nanocrystal films
}

\author{
Cooke, D. G.; Meldrum, A.; Jepsen, P. Uhd
}

Published in:

Applied Physics Letters

Link to article, DOI:

$10.1063 / 1.4767145$

Publication date:

2012

Document Version

Publisher's PDF, also known as Version of record

Link back to DTU Orbit

Citation (APA):

Cooke, D. G., Meldrum, A., \& Jepsen, P. U. (2012). Ultrabroadband terahertz conductivity of Si nanocrystal films. Applied Physics Letters, 101(21), 211107. https://doi.org/10.1063/1.4767145

\section{General rights}

Copyright and moral rights for the publications made accessible in the public portal are retained by the authors and/or other copyright owners and it is a condition of accessing publications that users recognise and abide by the legal requirements associated with these rights.

- Users may download and print one copy of any publication from the public portal for the purpose of private study or research.

- You may not further distribute the material or use it for any profit-making activity or commercial gain

- You may freely distribute the URL identifying the publication in the public portal

If you believe that this document breaches copyright please contact us providing details, and we will remove access to the work immediately and investigate your claim 


\section{AIP Appiled Physics Letters}

\section{Ultrabroadband terahertz conductivity of Si nanocrystal films}

D. G. Cooke, A. Meldrum, and P. Uhd Jepsen

Citation: Appl. Phys. Lett. 101, 211107 (2012); doi: 10.1063/1.4767145

View online: http://dx.doi.org/10.1063/1.4767145

View Table of Contents: http://apl.aip.org/resource/1/APPLAB/v101/i21

Published by the American Institute of Physics.

\section{Related Articles}

Tip-enhanced sub-diffraction fluorescence imaging of nitrogen-vacancy centers in nanodiamonds Appl. Phys. Lett. 102, 013102 (2013)

Acceptors in $\mathrm{ZnO}$ nanocrystals: A reinterpretation

Appl. Phys. Lett. 101, 262106 (2012)

Investigation of the thermal charge "trapping-detrapping" in silicon nanocrystals: Correlation of the optical properties with complex impedance spectra

Appl. Phys. Lett. 101, 242108 (2012)

Spectral patterns underlying polarization-enhanced diffractive interference are distinguishable by complex trigonometry

Appl. Phys. Lett. 101, 183104 (2012)

Near-infrared enhanced carbon nanodots by thermally assisted growth

Appl. Phys. Lett. 101, 163107 (2012)

\section{Additional information on Appl. Phys. Lett.}

Journal Homepage: http://apl.aip.org/

Journal Information: http://apl.aip.org/about/about_the_journal

Top downloads: http://apl.aip.org/features/most_downloaded

Information for Authors: http://apl.aip.org/authors

\section{ADVERTISEMENT}

\section{AIP Applied Physics Letters}

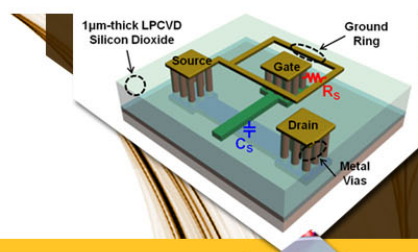

\section{SURFACES AND} INTERFACES

Focusing on physical, chemical, biological structural, optical, magnetic and electrical properties of surfaces and interfaces, and more..

\section{EXPLORE WHAT'S NEW IN APL}

SUBMIT YOUR PAPER NOW!
ENERCY CONVERSION AND STORACE 


\title{
Ultrabroadband terahertz conductivity of Si nanocrystal films
}

\author{
D. G. Cooke, ${ }^{1, a)}$ A. Meldrum, ${ }^{2}$ and P. Uhd Jepsen ${ }^{3}$ \\ ${ }^{1}$ Department of Physics, McGill University, Montreal, Quebec H3A 2T8, Canada \\ ${ }^{2}$ Department of Physics, University of Alberta, Edmonton, Alberta T6G 2M9, Canada \\ ${ }^{3}$ Department of Photonics Engineering, Technical University of Denmark, DK-2800 Kgs. Lyngby, Denmark
}

(Received 3 September 2012; accepted 29 October 2012; published online 21 November 2012)

\begin{abstract}
The terahertz conductivity of silicon nanoparticles embedded in glass with varying density is studied with ultra-broadband terahertz spectroscopy on picosecond time scales following fs optical excitation. The transition from relatively isolated charge carriers to densities which allow inter-particle transport is clearly observed. For the times immediately following carrier injection, we observe Drude-like long range transport that is rapidly replaced with a localized response on picosecond time scales. The localized response can be very well described by a phenomenological Drude-Smith model, verifying the applicability of this simple model to the conductivity of nanoparticle ensembles over the entire $\mathrm{THz}$ spectral window. (C) 2012 American Institute of Physics. [http://dx.doi.org/10.1063/1.4767145]
\end{abstract}

Semiconductor nanoparticles are attractive materials for tailoring the electronic and optical response of various optoelectronic devices. ${ }^{1}$ The motion of charge carriers in arrays of these materials is intricately related to the performance of these devices, and so understanding the transport in these systems is of vital importance. It is often challenging, however, to distinguish between intrinsic conduction effects and those related to contact issues. ${ }^{2}$ The non-contact, optical method of time-domain terahertz $(\mathrm{THz})$ spectroscopy has shown itself to be a valuable tool for probing ultrafast conductivity in bulk and nanoscale semiconductors and other materials. $^{3}$ The $\mathrm{THz}$ frequency probes $\mathrm{nm}$ scale motion of charge carriers, as the field reverses direction within a ps or less. Thus, THz spectroscopy is capable of unraveling intrinsic conduction mechanisms in nanostructures, in a timeresolved manner. In arrays of coupled nanoparticles and nanocrystalline films, the inter-particle conductivity was found to be sensitive to the volume fraction, essentially probing the percolation transition. ${ }^{4-13}$

In this work, we extend the bandwidth of previous studies from $3 \mathrm{THz}$ to $12 \mathrm{THz}$ and examine the ultrafast photoconductivity of $\mathrm{Si} / \mathrm{SiO}_{2}$ nanocomposite films for various $\mathrm{Si}$ nanoparticle concentrations. We clearly observe identifiable conductivity features in the spectrum associated with carrier localization. The smooth ac percolation transition is observed as the volume fraction of photoconductive Si nanoparticles embedded in insulating $\mathrm{SiO}_{2}$ is reduced, accompanied by a drop in the ac conductivity at a $\mathrm{Si}$ volume fraction $\rho_{\mathrm{Si}}=34 \%$. The decay of charge carriers is well described by a single exponential decay, identified as trapping at unpassivated $\mathrm{Si} / \mathrm{SiO}_{2}$ surface states. In the earliest times following photoexcitation, the conductivity is less suppressed at low frequencies (or longer length scales), explained by the development of Schottky-type barriers as carriers are trapped at the interfaces. ${ }^{12}$ In all cases, the conductivity spectra are well described by a phenomenological Drude-Smith model which has been shown as a simple but natural extension of

a)cooke@physics.mcgill.ca. the Drude model for materials with some measure of localization. ${ }^{14,15}$

Here, we investigate a set of samples comprised of $\mathrm{Si}$ nanocrystals formed by thermal decomposition of $\mathrm{SiO}_{x}$. The net $\mathrm{Si}$ nanocrystal volume fraction in these samples was varied from $16 \%$ to $80 \%{ }^{16}$ Details of the sample preparation are given in a previous publication. ${ }^{12}$ Briefly, $\mathrm{SiO}_{x}$ films were deposited on a fused silica substrate by simultaneous electron beam and thermal evaporation. Post-deposition annealing at $1100{ }^{\circ} \mathrm{C}$ for $1 \mathrm{~h}$ in a $95 \% \mathrm{~N}_{2}+5 \% \mathrm{H}_{2}$ atmosphere leads to the formation of well-defined crystalline $\mathrm{Si}$ nanocrystals whose size and spacing depend on the annealing temperature and Si content. The particle size distribution and crystallinity were studied previously by electron microscopy. ${ }^{5,16}$ The thickness of the films was $200 \mathrm{~nm}$ for $x \leq 0.8$ and $1 \mu \mathrm{m}$ for $x \geq 1.0$. The $\mathrm{O} / \mathrm{Si}$ ratios $x$ (Si volume fractions $\rho_{\mathrm{Si}}$ ) for the annealed $\mathrm{SiO}_{x}$ films investigated was $x=0.2$ (80\%), 0.4 (64\%), 0.6 (51\%), 0.8 (40\%), 1.0 (31\%), 1.2 $(23 \%)$, and $1.4(16 \%)$.

The time-resolved terahertz spectrometer is based on two-color laser plasma $\mathrm{THz}$ generation and an air-biased coherent detection scheme similar to that described in Ref. 17, producing a ultra-broadband $\mathrm{THz}$ pulses whose bandwidth is limited solely by the duration of the optical pulses. Details of the experimental setup can be found in Ref. 15. The $\mathrm{THz}$ pulses probe the conductivity of the sample in reflection geometry, collimated and focused on the sample surface, and then directed to a set of high voltage biased electrodes after reflection from the sample surface via a silicon beam splitter. This configuration avoids the strong absorption of the sample's $\mathrm{SiO}_{2}$ substrate. The $\mathrm{THz}$ pulse is detected by the method of air-biased coherent detection. ${ }^{17} \mathrm{~A}$ third portion of the fundamental beam is frequency doubled to $400 \mathrm{~nm}$ in a $\mathrm{BBO}$ crystal and used to photoexcite the samples at a pump fluence of $800 \mu \mathrm{J} / \mathrm{cm}^{2}$. Experiments are performed under a dry $\mathrm{N}_{2}$ purge to avoid absorption by water vapor.

The complex reflection coefficient is given by $\tilde{r}(\omega)=E_{\text {pump }}\left(\omega, \tau_{p}\right) / E_{\text {ref }}(\omega)$, the ratio of the Fourier transformed $\mathrm{THz}$ waveforms in the presence $\left(E_{\text {pump }}\right)$ and absence $\left(E_{\text {ref }}\right)$ of a pump pulse after pump-probe delay time $\tau_{p}$. The 

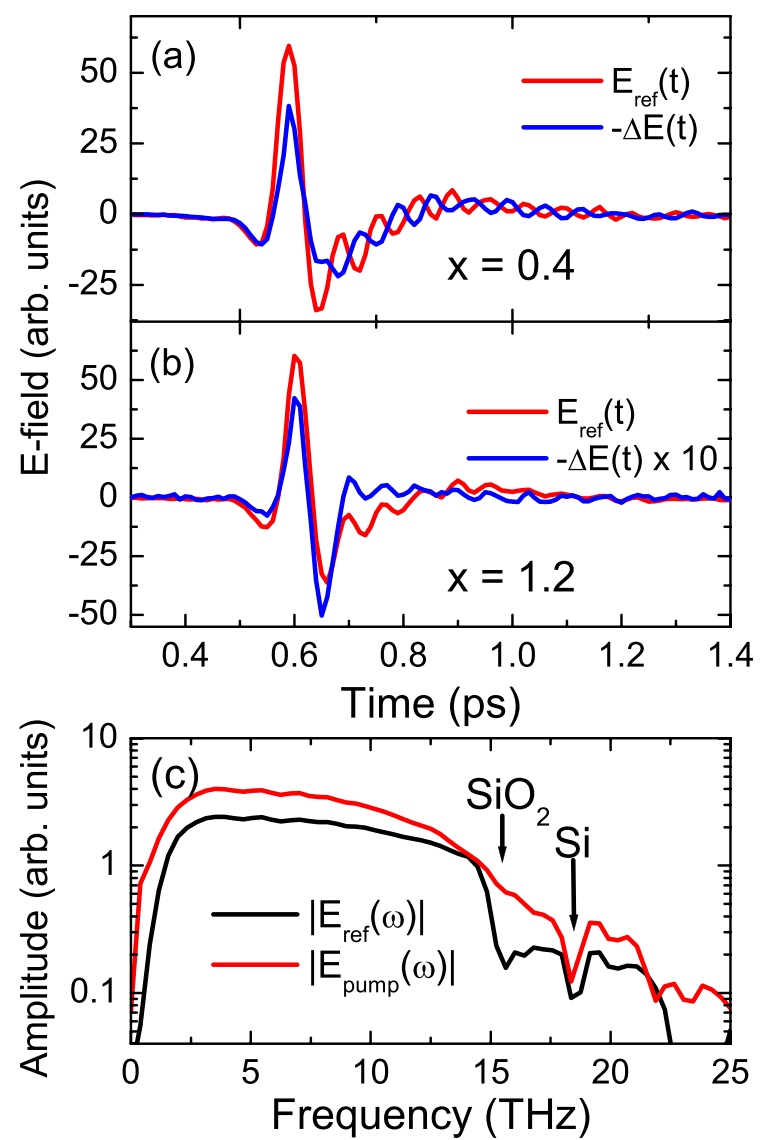

FIG. 1. Time domain reference and differential $-\Delta \mathrm{E}\left(\mathrm{t}, \tau_{\mathrm{p}}\right)=\mathrm{E}_{\mathrm{pump}}\left(\mathrm{t}, \tau_{\mathrm{p}}\right)$ $-\mathrm{E}_{\mathrm{ref}}(\mathrm{t})$ waveforms for the (a) $x=0.4$ and (b) $x=1.2, \mathrm{SiO}_{\mathrm{x}}$ nanocrystal films. (c) Fourier amplitude spectrum of the reflected $\mathrm{THz}$ pulse from the $x=0.4$ sample in the absence, $E_{r e f}(\mathrm{t})$ (black), and presence, $E_{\text {pump }}(\mathrm{t})$ (red), of the pump pulse 2 ps after $400 \mathrm{~nm}$ excitation.

transient pump-induced complex ac conductivity, $\tilde{\sigma}\left(\omega, \tau_{p}\right)$, of the Si nanocrystal composite film is related to $\tilde{r}\left(\omega, \tau_{p}\right)$ by inverting

$$
\tilde{r}\left(\omega, \tau_{p}\right)=\frac{1-\tilde{n}_{s}(\omega)-Z_{0} \tilde{\sigma}\left(\omega, \tau_{p}\right) d}{1+\tilde{n}_{s}(\omega)+Z_{0} \tilde{\sigma}\left(\omega, \tau_{p}\right) d}\left(\frac{1+\tilde{n}_{s}(\omega)}{1-\tilde{n}_{s}(\omega)}\right),
$$

where $\tilde{n}_{s}(\omega)$ is the frequency dependent index of refraction for the $\mathrm{SiO}_{2}$ substrate, $d$ is the thickness of the film, and $Z_{0}$ is the impedance of free space.

Representative differential, $-\Delta E(t)=E_{\text {pump }}(t)-E_{\text {ref }}(t)$, and $E_{\text {ref }}(t)$ waveforms for the $x=0.4$ and $x=1.2$ films recorded at a pump-probe delay of 2 ps are shown in Figs. 1(a) and 1(b), respectively. The sharp change in reflectivity of the substrate is responsible for the appearance of postpulse ringing in the time domain in the reference pulse at a frequency of $14.8 \mathrm{THz}$, indicated in the amplitude spectrum in Fig. 1(c). For the $x=0.4$ film, the differential transmission, $-\Delta E(t)$ has an out of phase, screening response of the substrate phonon whereas the $x=1.2$ film shows an in-phase post-pulse oscillation. This reflects the metallic nature of the higher Si content $x=0.4$ film where mobile carriers can screen the underlying phonon response of the substrate, as opposed to the $x=1.2$ film where carriers are primarily confined to their parent nanocrystals and are unable to screen the substrate.
The reflectivity of the amorphous $\mathrm{SiO}_{2}$ substrate in the $<20 \mathrm{THz}$ frequency range is dominated by the TO phonon related to rocking of bridging oxygen relative to the axis joining two neighboring $\mathrm{Si}$ atoms. ${ }^{18,19}$ The substrate complex index of refraction $\tilde{n}_{\mathrm{s}}(\omega)=n_{\mathrm{s}}(\omega)+i \kappa(\omega)$ was extracted using the reflection from the substrate referenced to a silver mirror. We must adjust the position of the sample reflection plane relative to the mirror by numerically adding $6 \mu \mathrm{m}$ to correct the phase response to recover the known low frequency $n_{\mathrm{s}}(\omega) .{ }^{20}$ After this procedure, the index was found to be in good agreement with previous measurements over the complete $\mathrm{THz}$ spectral range. ${ }^{21}$ The dispersion of the substrate results in a sharp change in the reflectivity in the 13-16 THz range that strongly influences the extracted conductivity, as can be seen in the amplitude spectra of the reference and pumped $x=0.4$ sample in Fig. 1(c). We model the dielectric function, $\tilde{\epsilon}_{\text {subs }}(\omega)=\tilde{n}_{\mathrm{s}}(\omega)^{2}$ of the substrate as a simple Lorentzian ${ }^{21} \tilde{\epsilon}_{\text {subs }}(\omega)=\epsilon_{\infty}+\omega_{0}^{2} /\left[\omega_{0}^{2}-\omega^{2}+I \omega \Gamma\right]$ with $\epsilon_{\infty}=2.86, \omega_{0} / 2 \pi=13.4 \mathrm{THz}, \quad$ and $\Gamma=1.4 \mathrm{THz}$. Additionally there is an absorption line at $18.8 \mathrm{THz}$ due to the Si beamsplitter, resulting from the TO + TA phonon sum mode, indicated by an arrow in Fig. 1(c).

The $\tilde{\sigma}\left(\omega, \tau_{p}\right)$ spectra recorded 2 ps following photoexcitation and are shown in Fig. 2 for the entire $x$ series. The conductivity is normalized to the maximum real conductivity in the spectral band, $\sigma_{\max }$, which is indicated in the respective plots. One can observe several features as the Si volume fraction is reduced. At the highest Si content of $x=0.2$, the spectrum is very similar to a simple Drude response, which would be expected for a homogeneous Si film. With decreasing $\mathrm{Si}$ content, the low frequency $\sigma_{1}(\omega)$ spectra are suppressed and the spectral weight is shifted to higher frequency. Concomitantly, the low frequency $\sigma_{2}(\omega)$ spectrum moves from an inductive response (positive) to capacitive response (negative), with the crossover moving to higher frequency. The magnitude of the conductivity response is also strongly reduced as the volume of $\mathrm{Si}$ decreases, as expected. Due to the sharp drop of the substrate reflection in the $13-15 \mathrm{THz}$ range, the conductivity exhibits artifacts near $12 \mathrm{THz}$ for the lowest conductive samples.

As in previous low frequency work between 0.3 and 2.5 $\mathrm{THz}$, the $\tilde{\sigma}(\omega)$ response can be well described by the phenomenological Drude-Smith model given by

$$
\tilde{\sigma}(\omega)=\frac{\omega_{p}^{2} \epsilon_{0} \tau}{1-i \omega \tau}\left[1+\sum_{n=0}^{\infty} \frac{\mathrm{c}_{n}}{(1-i \omega \tau)^{n}}\right],
$$

where $\omega_{p}$ is the plasma frequency, $\epsilon_{0}$ is the permittivity of free space, $\tau$ is the momentum scattering time, and $c_{n}$ are socalled back-scattering coefficients. As reported recently, ${ }^{15}$ the $c$ parameters can be viewed merely as the Taylor coefficients of the expanded multiplicative function that describes the deviation from a Drude exponentially decaying current response function. Thus, taking only the first term, we can view this as a first order correction of the conductivity response due to the localization in the $\mathrm{Si}$ nanoparticles and we drop the subscript $n$ in the following discussion.

Figure 3 shows the Drude-Smith fit parameters for two representative films in the series, $x=0.6$ and 1.2. The square of the plasma frequency $\omega_{p}^{2}$ is proportional to the carrier 


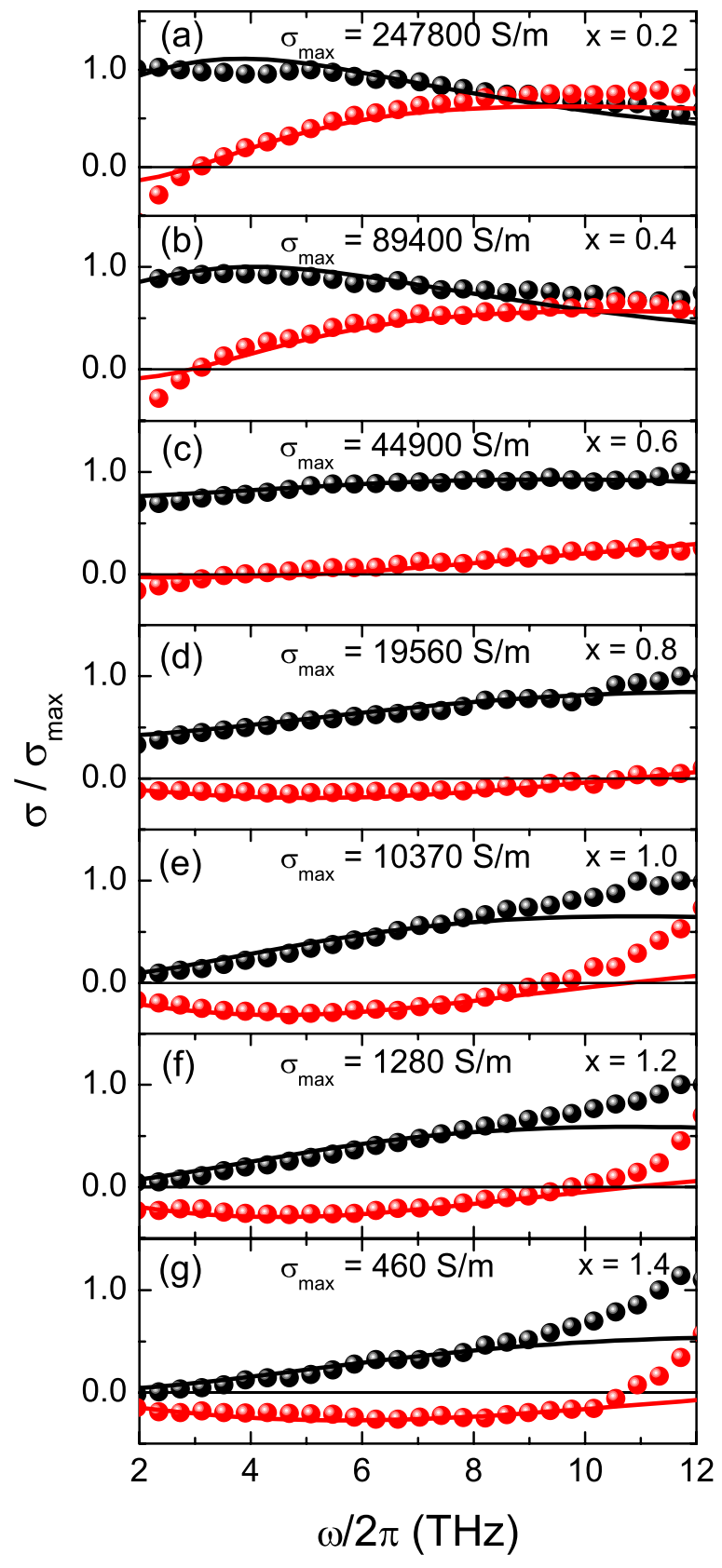

FIG. 2. Real (black dots) and imaginary (red dots) components of the conductivity spectrum recorded $2 \mathrm{ps}$ after $400 \mathrm{~nm}$ photoexcitation of $\mathrm{SiO}_{x}$ nanocrystal films with (a)-(g) $x=0.2-1.4$, respectively. Black and red lines are best fits from the Drude-Smith model, described in the text.

density and is plotted in Fig. 3(a). The carrier dynamics follow a clear single exponential decay with recombination times of $39 \pm 1 \mathrm{ps}$ for $x \leq 1.0$ and increasing to $220 \pm 15 \mathrm{ps}$ for $x>1.0$. This rapid increase in carrier lifetime can be explained by the breakup of percolation paths as the volume fraction of photoconductive silicon dips below the metalinsulator transition, as carriers remain isolated on their parent nanocrystal and cannot reach distributed trap states. The carrier scattering time is shown in Fig. 3(b), where one can see an obvious increasing trend for films above the percolation transition with time that has not been previously observed. Expressing $\tau$ as a mean free path $\ell_{m f}=v_{t h} \tau$ using a thermal velocity $v_{t h}=\sqrt{3 \mathrm{k}_{\mathrm{B}} \mathrm{T} / m^{*}}=2.3 \times 10^{5} \mathrm{~m} / \mathrm{s}$, assuming an $m^{*}=0.26 m_{e}$ and $\mathrm{T}=300 \mathrm{~K}, \ell_{m f}$ increases linearly from $3 \mathrm{~nm}$, the size of the nanocrystals, to $10 \mathrm{~nm}$, close to the
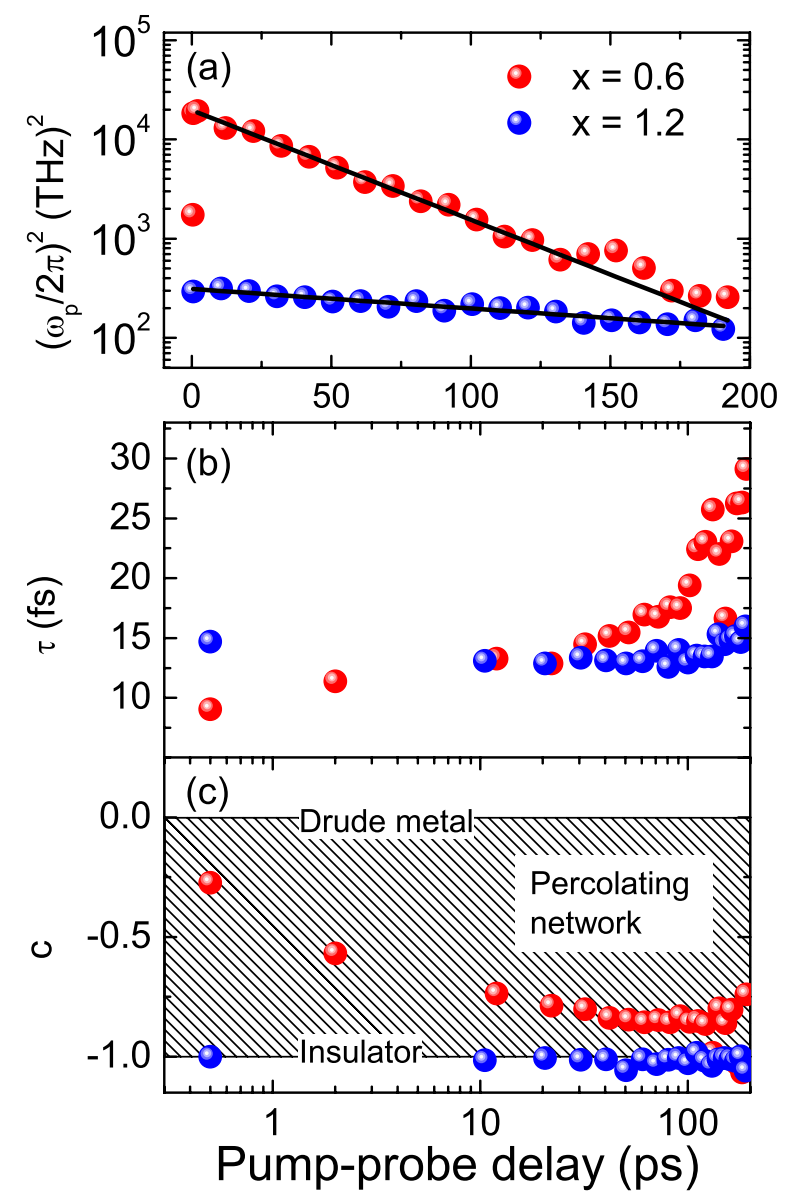

FIG. 3. Dynamics of the (a) square of the plasma frequency, (b) scattering time, and (c) back-scattering parameter $c$ extracted from Drude-Smith model fit parameters to the complex conductivity data for the $x=0.6$ (red dots) and $x=1.2$ (blue dots) films.

bulk Si electron mean free path at comparable charge carrier densities of $10^{18}-10^{19} \mathrm{~cm}^{-3}$ where the electron mobility is approximately $300 \mathrm{~cm}^{2} / \mathrm{Vs}^{22}$ Due to quantum confinement of the smallest particles, it is energetically favorable for charges to migrate to the larger islands. However, below the percolation transition, charges are localized to their parent particles which have a set size distribution and the scattering time is set by the mean particle diameter. ${ }^{12}$

The backscatter coefficient, $c$, is shown in Fig. 3(c). The higher Si content $x=0.6$ film shows Drude-like behavior $(c=-0.27)$ at a pump-probe delay of $500 \mathrm{fs}$ after carrier injection. A $c$ parameter becoming more negative with increasing pump-probe delay was previously observed by Titova et al. and explained by the establishment of Schottkytype barriers as carriers become trapped at the surfaces of the nanoparticles. ${ }^{12}$ Such a mechanism is also consistent with an increasing $\tau$ after excitation, due to removal of final scattering states via Pauli blocking, but inconsistent with a model of diffusion limited trapping.

A measure of the total spectral weight for the entire series at early times $\left(\tau_{p}=2 \mathrm{ps}\right.$ ) can be obtained by integrating $\sigma_{1}(\omega)$ over the wide range between 2 and $9 \mathrm{THz}$, as plotted in Fig. 4. With changing $\rho_{\mathrm{Si}}$, one can see a clear change in the decreasing spectral weight occurring at $\rho_{\mathrm{Si}}=34 \%$ above which the film is metallic and below insulating, consistent with the theoretical 3D percolation transition $\left(\rho_{\mathrm{Si}}=33 \%\right)$ 


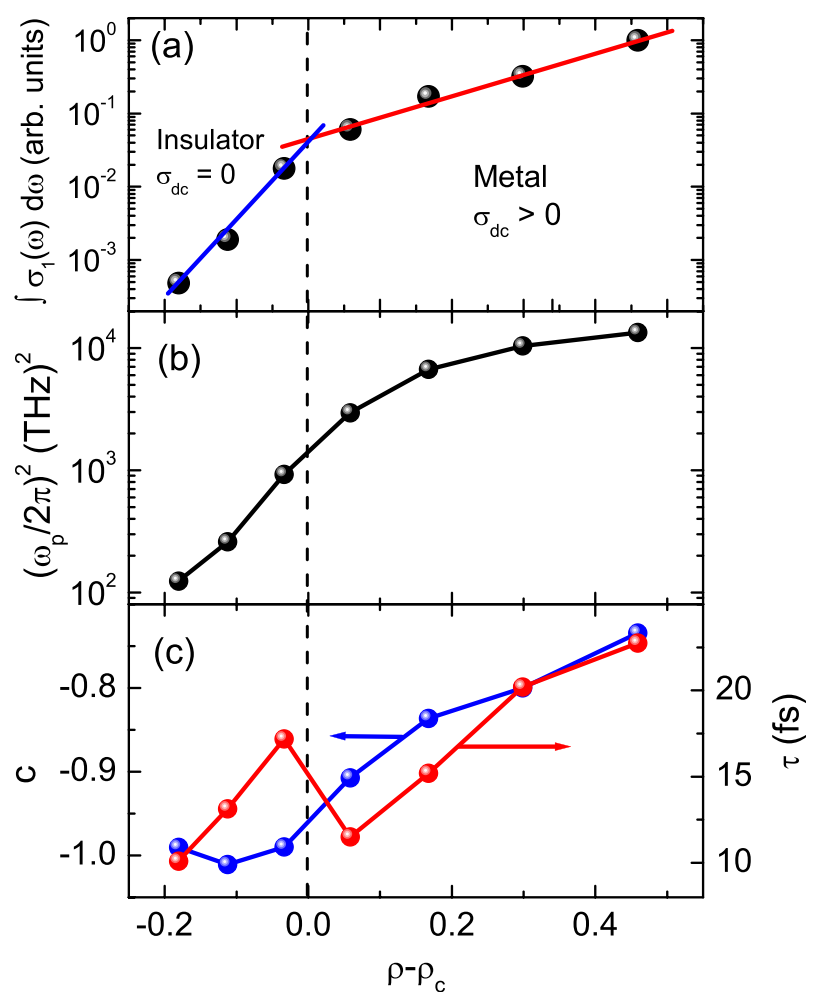

FIG. 4. (a) Integrated $\sigma_{1}(\omega)$ between the frequency interval 2-9 THz demonstrating the percolation transition at the critical volume fraction $\rho_{c}=34 \%$. The Drude-Smith fit parameters for pump-probe delay of 40 ps: (b) squared plasma frequency, (c) backscatter parameter $c$ and scattering time $\tau$.

and in good agreement with previous steady state transport $^{23,24}$ and previous THz work. ${ }^{12}$ Taking a cross-section of the data series at $\tau_{\mathrm{p}}=40 \mathrm{ps}$ and examining the Drude-Smith fit parameters, we see that the carrier density proportional to $\omega_{p}^{2}$ in Fig. 4(b) shows no deviation from a smoothly decreasing trend at $\rho_{\mathrm{c}}$, whereas in Fig. 4(c), the $c$ parameter marks the transition by reaching an asymptotic value of -1 (localized). Also $\tau$ shows a sharp increase consistent with observations by Walther et al. for thin gold films near the critical percolation film thickness. ${ }^{8}$ This behavior was linked to the expected divergence of the real dielectric function $\epsilon_{1}(\omega),{ }^{25}$ which can be thought of as the capacitance of the nanoparticles having a divergence the moment an insulating barrier develops between conducting islands. Since $\tau \propto \omega \epsilon_{1}(\omega) / \sigma_{1}(\omega)$, a divergence is expected if $\omega \epsilon_{1}$ increases faster than $\sigma_{1}$ decreases as $\rho_{c}$ is approached.

In summary, we have measured the ultrafast $\tilde{\sigma}(\omega)$ dynamics of a series of $\mathrm{Si} / \mathrm{SiO}_{2}$ nanocomposites above and below the percolation transition. The $\mathrm{THz}$ conductivity is found to be well described by the Drude-Smith model over the complete spectral range and the decay of mobile carriers is due to a single decay channel consistent with trapping at unpassivated surface states. ${ }^{5}$ The localization of carriers occurs dynamically on a several tens of picoseconds time scale in films with $\rho_{S i} \geq \rho_{c}$. The scattering time is found to increase significantly with delay time for films above $\rho_{c}$ and constant below $\rho_{c}$, a signature of inter-particle diffusion disappearing in the insulating phase. Finally an increase in the scattering time is observed at $\rho_{c}$ and we tentatively link this with a divergence of the dielectric function at $\rho_{c}$.

The authors acknowledge financial support from NSERC, CFI, FRQNT, the H. C. Ørsted fund and the Danish Council for Independent Research I Technology and Production Sciences (HI-TERA Project).

${ }^{1}$ N. Daldosso and L. Pavesi, Laser Photonics Rev. 3, 508 (2009).

${ }^{2}$ P.-E. Trudeau, M. Sheldon, V. Altoe, and A. P. Alivisatos, Nano Lett. 8, 1936 (2008).

${ }^{3}$ P. U. Jepsen, D. G. Cooke, and M. Koch, Laser Photonics Rev. 5, 124 (2011).

${ }^{4}$ M. C. Beard, G. M. Turner, J. E. Murphy, O. I. Micic, M. C. Hanna, A. J. Nozik, and C. A. Schmuttenmaer, Nano Lett. 3, 1695 (2003).

${ }^{5}$ D. G. Cooke, A. N. MacDonald, A. Hryciw, J. Wang, Q. Li, A. Meldrum, and F. A. Hegmann, Phys. Rev. B 73, 193311 (2006).

${ }^{6}$ J. B. Baxter and C. A. Schmuttenmaer, J. Phys. Chem. B 110, 25229 (2006).

${ }^{7}$ D. G. Cooke, A. N. MacDonald, A. Hryciw, A. Meldrum, J. Wang, Q. Li, and F. A. Hegmann, J. Mater. Sci.: Mater. Electron. 18, 447 (2007).

${ }^{8}$ M. Walther, D. G. Cooke, C. Sherstan, M. Hajar, M. R. Freeman, and F. A. Hegmann, Phys. Rev. B 76, 125408 (2007).

${ }^{9}$ H. Němec, P. Kuzel, and V. Sundström, Phys. Rev. B 79, 115309 (2009).

${ }^{10}$ H. Nĕmec, P. Kuzel, F. Kadlec, D. Fattakhova-Rohlfing, J. Szeifert, T. Bein, V. Kalousek, and J. Rathousky, Appl. Phys. Lett. 96, 062103 (2010).

${ }^{11}$ H. Nĕmec, J. Rochford, O. Taratula, E. Galoppini, P. Kuzcaronel, T. Polívka, A. Yartsev, and V. Sundström, Phys. Rev. Lett. 104, 197401 (2010).

${ }^{12}$ L. V. Titova, T. L. Cocker, D. G. Cooke, X. Wang, A. Meldrum, and F. A. Hegmann, Phys. Rev. B 83, 085403 (2011).

${ }^{13}$ Z. Mics, H. Němec, I. Rychetský, P. Kuzel, P. Formánek, P. Malý, and P. Nĕmec, Phys. Rev. B 83, 155326 (2011).

${ }^{14}$ N. V. Smith, Phys. Rev. B 64, 155106 (2001).

${ }^{15}$ D. G. Cooke, F. C. Krebs, and P. U. Jepsen, Phys. Rev. Lett. 108, 056603 (2012).

${ }^{16}$ J. Wang, X. F. Wang, Q. Li, A. Hryciw, and A. Meldrum, Philos. Mag. 87, 11 (2007)

${ }^{17}$ I.-C. Ho, X. Guo, and X.-C. Zhang, Opt. Express 18, 2872 (2010).

${ }^{18}$ M. K. Gunde, Physica B 292, 286 (2000).

${ }^{19}$ A. Sassella, A. Borghesi, F. Corni, A. Monelli, G. Ottaviani, R. Tonini, B. Pivac, M. Bacchetta, and L. Zanotti, J. Vac. Sci. Technol. A 15, 377 (1997).

${ }^{20}$ D. Grischkowsky, S. Keiding, M. van Exter, and C. Fattinger, J. Opt. Soc. Am. B 7, 2006 (1990).

${ }^{21}$ R. Kitamura, L. Pilon, and M. Jonasz, Appl. Opt. 46, 8118 (2007).

${ }^{22}$ C. Jacoboni, C. Canali, G. Ottaviani, and A. Alberigi Quaranta, Solid-State Electron. 20, 77 (1977).

${ }^{23}$ I. Balberg, E. Savir, J. Jedrzejewski, A. G. Nassiopoulou, and S. Gardelis, Phys. Rev. B 75, 235329 (2007)

${ }^{24}$ I. V. Antonova, M. B. Gulyaev, Z. S. Yanovitskaya, V. A. Volodin, D. V. Marin, M. D. Efremov, Y. Goldstein, and J. Jedrzejewski, Semiconductors 40, 1198 (2006).

${ }^{25}$ M. Hövel, B. Gompf, and M. Dressel, Phys. Rev. B 81, 035402 (2010). 